\title{
Biotoxicity of Cry1Ab protein on wolf spider Pardosa pseudoannulata
}

\author{
Juan Wang ${ }^{1}$ Yuande Peng ${ }^{2}$ Huilin Yang ${ }^{1}$ Zhenzhen Yan ${ }^{1}$ Qinjin Li ${ }^{1} \cdot$ Yixue Shi $^{1}$ • \\ Chunliang $\mathrm{Xie}^{2} \cdot$ Yunshan Liang $^{1} \cdot$ Zhi Wang $^{1} \cdot$ Qisheng Song $^{3}$
}

Accepted: 13 September 2017 / Published online: 17 October 2017

(C) The Author(s) 2017. This article is an open access publication

\begin{abstract}
In this research, we carried out a tritrophic bioassay to assess the potential effect of Cry1 Ab-expressing rice on the foraging behavior of the common wolf spider Pardosa pseudoannulata and its underlying molecular mechanism. Results indicated the Bt-containing spiders expressed a higher foraging range when compared to controls. The high throughput de novo transcriptome sequencing was further carried out for central nervous system (CNS) of P. pseudoannulata with and without Cry1 Ab intake. We obtained 120, 985 unigenes with a mean length of $529.73 \mathrm{bp}$. Functional analysis of differentially expressed genes (DEGs) showed the expression levels of genes related to energy metabolism were changed in response to Cry $1 \mathrm{Ab}$, which may contribute to a more active foraging behavior. In addition, some DEGs also have a function related to metal ion binding, implying a potential influence on metal ionsdependent reactions. This may be associated with Cry $1 \mathrm{Ab}$ resistance mechanism in the spider.
\end{abstract}

Electronic supplementary material The online version of this article (https://doi.org/10.1007/s10646-017-1858-4) contains supplementary material, which is available to authorized users.

Juan Wang, Yuande Peng and Huilin Yang are co-first author.

Zhi Wang

wangzhispider@hotmail.com

1 College of Bioscience and Technology, Hunan Agriculture University, No1 Nongda Road, Changsha 410128 Hunan, China

2 Institute of Bast Fiber Crops, Chinese Academy of Agricultural Sciences, Changsha, Hunan 410205, China

3 Division of Plant Sciences, University of Missouri, Columbia, MO 65211, USA
Keywords $\mathrm{Cry} 1 \mathrm{Ab} \cdot \mathrm{CNS} \cdot$ behavior $\cdot$ transcriptome $\cdot$ P. pseudoannulata

\section{Introduction}

Spiders are effective predators in terrestrial ecosystems, where they act in the ecology of herbivore and detritivore food webs (Jung et al. 2008a) and influence herbivore populations (Marc and Ysnel 1999). Spider species richness also is used in ecological conservation and assessment of environmental changes (Duelli 1998; Sauberer et al. 2004). The common wolf spider Pardosa pseudoannulata (Bosenberg et Strand) (Araneae: Lycosidae) is a major predator in Chinese agroecosystems (Tian et al. 2012). It is a biological control agent of some insect pests, especially the brown planthopper (BPH), Nilaparvata lugens (Hemiptera: Delphacidae), a severe rice pest in China (Zhao and Chen 2004; Zhao and Zhang 2005).

Rice, Oryza sativa, is a major staple food for more than half of the world's population (Ouyang et al. 2005). However, the rice industry has been increasingly damaged by rice pests in the past few years (Bottrell 2012). Various traditional and biotechnology approaches have been employed to develop durable resistance to diseases and serious pests (Khush 2005). Genetically modified (GM) rice expressing the Bacillus thuringiensis (Bt) Cry1 Ab protein has been developed to alleviate the environmental contamination due to the use of broad-spectrum chemical insecticides and to address the broader complex of insect pests (Chen et al. 2011).

Because the biotechnology of creating and deploying $B t$ rice is available, studies were launched to thoroughly investigate the agroecological and environmental safety of $B t$ rice. Akhtar et al. (2010) evaluated the non-target impact 
of Bt rice on the thrips, Stenchaetothrips biformis, showing longer larval and pupal development and previposition durations, but shorter oviposition period and female adult longevity and fewer total laid eggs of the thrip when fed on $B t$ rice in comparison to non- $B t$ controls (Akhtar et al. 2010). Chen et al. (2009) indicated that $B t$ rice did not influence survivorship and fecundity of Pirata subpiraticus but contribute to a longer developmental time of spiderlings (Chen et al. 2009). Lee et al (2014) indicated that the transgenic Cry1Ac rice had no adverse effects on the spider community structure of the rice fields (Lee et al. 2014). Lu et al (2014) assess the potential adverse effects of $B t$ rice on the non-target herbivore, Nephotettix cincticeps, and found there were no significant differences in some ecological fitness parameters including egg development duration, fresh adult weight, adult longevity, and oviposition period (Lu et al. 2014). However, the influence of Bt rice on behavioral ecology and its underlying molecular mechanisms in non-target organisms remain unexplored. In the present study, we performed transcriptomic analysis of CNSs isolated from control spiders maintained on nontransgenic rice and from experimental spiders taken from $B t$ rice, and identified differentially expressed genes (DEGs). We also recorded the influence of $B t$ on foraging behavior of spider under laboratory conditions, and found $B t$ rice caused a more active foraging behavior of the spider.

\section{Materials and methods}

\section{Rice sample preparation}

The seeds of the transgenic $B t$ Shanyou 63 rice, expressing a Cry1 Ab gene, and its non-transgenic parental commercial cultivar Shanyou 63 were provided by Life Science College, Hunan Normal University. Both rice lines were grown in the experimental farmland at Hunan Agricultural University $\left(113.08^{\circ} \mathrm{E}, 28.18^{\circ} \mathrm{N}\right)$. They were individually potted and cultured under the nylon nets $(175 \times 185 \times 225 \mathrm{~cm})$ to preclude extrinsic interference, such as unrelated insects and predators from the neighboring fields. No insecticides were sprayed in or near the field during the experimental period.

\section{Animal sample preparation}

Laboratory brown planthopper (BPH) colonies were established from the experimental field of Hunan Agricultural University. Each colony was reared on either Cry1 Ab expressing (experimental) or control rice. BPHs were separately taken from both rice lines after 15 days continuous feeding, then provided to spiders as prey (Tian et al. 2013).

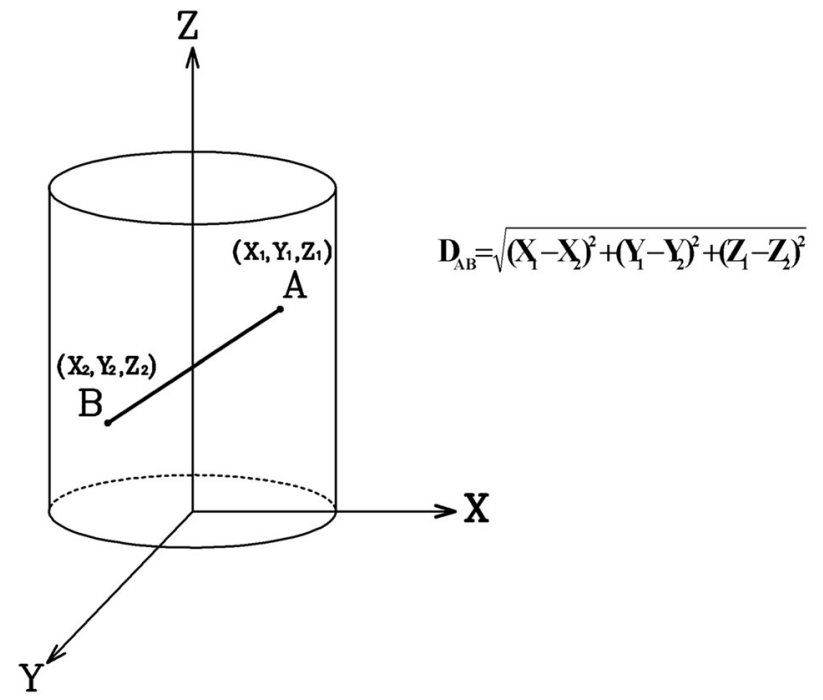

Fig. 1 The foraging displacements of P. pseudoannulata formed in a three-dimensional coordinate system. A spider was captured at the position $\mathrm{A}(\mathrm{X} 1, \mathrm{Y} 1, \mathrm{Z} 1)$ and $\mathrm{B}(\mathrm{X} 2, \mathrm{Y} 2, \mathrm{Z} 2)$ every ten seconds. A unit of foraging displacement was recorded by calculating the two ordinates absolute distance

Adult female spiders ( $P$. pseudoannulata) were collected from the organic farmlands of Hunan Academy of Agriculture Sciences and each spider was kept in a separate glass tube ( $15 \mathrm{~mm}$ diameter, $100 \mathrm{~mm}$ height). Each tube was topped with cotton balls and the inner tube wall was covered with a piece of soaked cotton to maintain humidity. Collected spiders were assorted into two groups (experimental and control) and starved for $48 \mathrm{~h}$ before being supplied each with 20 BHPs from experimental or control rice per day. All the tubes were numbered and remained in a temperature-controlled chamber $\left(25 \pm 2{ }^{\circ} \mathrm{C}, 70 \pm 10 \% \mathrm{RH}\right.$ and 16 L:8D photoperiod; the photophase was from 06:00 to 22:00). In addition, our previous study indicated that accumulated Cry $1 \mathrm{Ab}$ level in spider peaked at 9th day after spider was fed with $B t$ rice-consuming BHPs (Wang et al. 2016), so spiders used in this research were all fed with $B t$ containing BPHs for 9 days. Spiders were then not given food for $48 \mathrm{~h}$ before testing.

\section{Effect of Cry1Ab rice on the foraging behavior of $P$. pseudoannulata}

Fifteen spider adults of similar size (five spiders per repetition) were selected from the test and control groups respectively, transferred to individual cylinders $(36.8 \mathrm{~cm}$ height, $6.5 \mathrm{~cm}$ diameter). The bottom of each cylinder was covered with moist mud $(3 \mathrm{~cm}$ height $)$ at the farmland of Hunan Agriculture University and a rice stem $30 \mathrm{~cm}$ height) provided by Hunan Academy of Agriculture Sciences, was vertically placed in the mud to simulate the farmland environment. Each cylinder was provided with 
200 non Bt-rice reared BPHs and sealed with triple layers of gauze. The experiment was conducted in the temperaturecontrolled chamber as described and the foraging behavior was recorded by video camera for $48 \mathrm{~h}$. The following periods (3rd-4th hour; 28th-29th hour; 35th-36th hour) from the treated and control groups were selected for data collection.

The movement behavior of the spider was recorded and analyzed using the following method: we marked the position of the spider in the cylinder every $10 \mathrm{~s}$ through video replaying. And the linear distance between two continuous points was calculated by means of Pythagorean proposition, which represented the movement distance of spider per $10 \mathrm{~s}$ (Fig. 1). We divided the range of moving distance of the spider into five categories, including $0-3 \mathrm{~cm}$, $3-6 \mathrm{~cm}, 6-9 \mathrm{~cm}, 9-12 \mathrm{~cm}$, and $>12 \mathrm{~cm}$. Then the occurrence frequency of each moving range was calculated during $1 \mathrm{~h}$ period.

\section{Transcriptomic analysis}

\section{RNA isolation and sequencing}

CNSs were isolated from experimental and control spiders at $4{ }^{\circ} \mathrm{C}$, transferred to $1.5 \mathrm{ml}$ Eppendorf tubes, frozen with liquid nitrogen and stored at $-20^{\circ} \mathrm{C}$. RNA extraction and sequencing was performed by Oebiotech Enterprise, Shanghai. In brief, total RNA was extracted from central nervous system (CNS) of spider, using TRIzol (Invitrogen Corp, USA) according to the manufacturer's instructions. The integrity and quality of total RNA were checked using a NanoDrop ND-1000 spectrophotometer (NanoDrop Technologies Inc, Rockland, DE, USA), limiting the standard to $1.8 \leq$ OD260/OD280 $\leq 2.1$ and further confirmed by agarose gels.

RNA sequencing libraries were constructed and sequenced on an Illumina Hiseq 2000. Clean reads were assembled using the de novo transcriptome assembler Trinity after removing adaptor sequences, and low-quantity reads (reads with ambiguous bases "N" and duplicate sequences). Trinity combines relatively short, clean reads into longer continuous sequences, contigs, and assembles the contigs into longer unigenes. The unigenes were processed to remove redundancy, leading to non-redundant unigenes, which were used in the bioinformatics analysis reported here.

\section{Gene annotation and expression}

All unigenes ( $>200 \mathrm{bp}$ ) were analyzed against public databases (Nr/NCBI, SwissProt/UniProtKB, KOG/NCBI, KEGG, GO) using BLAST algorithm to understand gene biological functions. Three arthropod species databases
(Tetranychus urticae, Drosophila melanogaster, and Stegodyphus mimosarum) were interrogated to assess identity of the obtained unigenes (e-values $<1 \mathrm{e}-5$ ).

Expression of all unigenes in the spiders fed with test and control BPHs were estimated by calculating read density as 'reads per kilobase of exon model per million map reads' (RPKM) (Mortazavi et al. 2008). Employing the method by Audic and Claverie (1997), a strict algorithm was developed to identify differentially expressed genes (DEGs) between two libraries. A threshold false discovery rate of $<0.01$ and absolute value of $\log _{2}$ Foldchange $>2$ were used to determine significant differences in gene expression.

\section{$q P C R$}

To verify annotations of a set of selected genes, total RNA was isolated from each sample using TRIzol (Invitrogen Corp, USA) and treated with DNase I (Fermentas, Lithuania) according to the manufacturer's protocol. The cDNA was synthesized using a RevertAid ${ }^{\mathrm{TM}} \mathrm{H}$ Minus First Strand cDNA Synthesis Kit (Fermentas, Lithuania) and qPCR was performed using ABI 7900HT (ABI, USA) with a reaction volume of $25 \mu \mathrm{l}$, containing $1 \mu \mathrm{l}$ of 1:10 cDNA diluted with $\mathrm{ddH}_{2} \mathrm{O}, 12.5 \mu \mathrm{l} 2 \mathrm{x}$ SYBR Green Master Mix (ABI, USA) and $200 \mathrm{nM}$ of primers. Reactions were performed in triplicate to ensure consistent technical replication and run in 96-well plates under the following conditions: $94^{\circ} \mathrm{C}$ for $3 \mathrm{~min}$. and then followed by 40 cycles for $94{ }^{\circ} \mathrm{C}$ for $30 \mathrm{~s}$, $59^{\circ} \mathrm{C}$ for $30 \mathrm{~s}, 72{ }^{\circ} \mathrm{C}$ for $45 \mathrm{~s}$. Cycling ended at $72{ }^{\circ} \mathrm{C}$ for $5 \mathrm{~min}$. Relative gene expression was evaluated with a DataAssist Software version 3.0 (Applied Biosystems/Life Technologies), using $18 \mathrm{~s}$ rRNA as a reference gene for RNA load and gene expression in analyses. The relative quantitative method $\left(\Delta \Delta \mathrm{C}_{\mathrm{T}}\right)$ was used to calculate the fold change of target genes (Livak and Schmittgen 2001).

\section{Statistical analysis}

The data from foraging behavior and qPCR were analyzed using one-way analysis of variance (ANOVA) with SPSS 17.0 software. Significant differences at $p<0.05$ or $p<0.01$ are designated with $*$ or $* *$.

\section{Results}

Effects of Cry1Ab protein on the foraging behavior of P. pseudoannulata

Control and experimental spiders were provided abundant control BPHs $(n=200)$. During an hour time-period, all recorded displacements were categorized into five distance zones $(0-3,3-6,6-9,9-12,>12 \mathrm{~cm})$. The foraging range of 


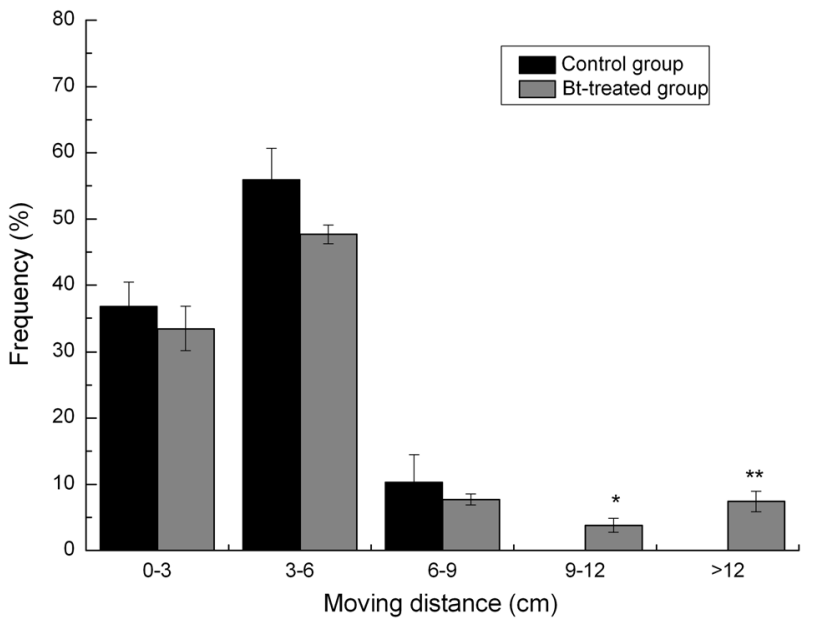

Fig. 2 The total foraging displacements of P. pseudoannulata on the different movement range of rice within $1 \mathrm{~h}$. The horizontal axis represents range of moving distance of spider per $10 \mathrm{~s}$. The vertical line represents the occurrence frequency of each moving range during an hour. Mean $( \pm \mathrm{SE})$ followed by asterisk $(*)(* *)$ in the figure were significantly different (One-way ANOVA, $p<0.05, p<0.01$ )

P. pseudoannulata on a rice stem, as a main parameter, reflected their foraging capability. Spiders foraged most frequently at the $3-6 \mathrm{~cm}$ range, both in test and control groups, followed by $0-3 \mathrm{~cm}$. Significant differences in the foraging range greater than $9 \mathrm{~cm}(p<0.01)$ were observed between the test and control spiders (Fig. 2).

\section{De novo assembly of all unigenes}

The spider CNS cDNA sample was prepared and sequenced using the Illumina Hiseq 2000 platform. We obtained nearly 95 million total raw reads, which was submitted to the SRA database (accession number: SRR 2010603, SRR 2010603). Finally, a total of 120, 985 unigenes were obtained, using Trinity software (Table 1). The mean unigene size is 529.73 bp, with lengths ranging from 201 to $34055 \mathrm{bp}$. The size distribution of these unigenes is shown in Figure S1.

\section{Functional annotation}

To date there remains no published complete reference genome for P. pseudoannulata. We put our sequences of CNS into five public databases and three arthropod databases as described in material and method section. Among all 120,985 unigenes, 34,081 unigenes $(28.17 \%$ of the total) were matched into $\mathrm{Nr}$ database, 29,472 unigenes (24.36\% of the total) to Swissprot database, 27,234 unigenes $(22.51 \%$ of the total) to COG, 29,194 unigenes ( $24.13 \%$ of the total) to GO database and 5,493 unigenes (4.54\% of the total) to KEGG database. And 7,042 unigenes (5.82\% of the total) showed similar sequences with the genome of Tetranychus urticae, 9,829 unigenes $(8.12 \%$ of
Table 1 Summary for the $P$. pseudoannulata CNS transcriptome

\begin{tabular}{ll}
\hline Total mumber of the raw reads & $94,971,094$ \\
Total base pairs(bp) & $9,592,080,494$ \\
Total number of transcript & 176251 \\
Total number of unigenes & 120985 \\
Mean length of ungenes & 529.73 \\
N50 & 661 \\
\hline
\end{tabular}

the total) with the genome of Drosophila melanogaster and 9,323 unigenes $(7.71 \%$ of the total) with the genome of Stegodyphus mimosarum.

Overall, 27,234 unigenes were functionally classified into 25 COG categories (Fig. 3). Among them, the largest category was "Signal transduction mechanisms" (9864; $36.22 \%$ ) followed "General function prediction only" (9399; $34.51 \%)$ and "Posttranslational modification, protein turnover, chaperones" (4581; 16.82\%), while the categories with the least representation were "Cell motility" $(125 ; 0.46 \%)$ and "Nuclear structure" (249;0.91\%).

The GO project provides an international standardized gene functional classification system for genes and gene products across all species. The ontology covers three domains: cellular component, molecular function and biological process (Ashburner et al. 2000). The unigenes assigned to the GO database were divided into three gene ontology classes: molecular function, cellular component and biological process. Among them, biological process made up the majority (64.49\%), followed by molecular function $(25.5 \%)$ and cell component $(10.01 \%)$. The largest categories in gene ontology classes, "biological process", "molecular function", and "cell component" were "integral component of membrane", "zinc ion binding", and "oxidation-reduction process" respectively (Fig. 4).

We conducted a blast search against the KEGG database for the assembled unigenes. Results showed that 5,493 unigenes had significant matches in the database and were assigned into 327 KEGG pathways (Table S1). The pathway most represented by the unique sequences was "Ribosome", "RNA transport" and "protein processing in endoplasmic reticulum".

\section{Functional analysis of DEGs}

In all, the expression of 42 unigenes was significantly changed in Cry1Ab-treated spiders, compared to controls (FDR < 0.01, Log ratio $>2$, Table S2). Forty-two GO terms were annotated by DEGs, which could be divided into three parts including energy, binding and others (Fig. 5a). In specific, 14 $\mathrm{GO}$ categories were acting in regulating energy metabolism in intracellular, such as "oxidative phosphorylation" (GO: 0006119), "cytochrome-c oxidase activity" (GO:0004129), 


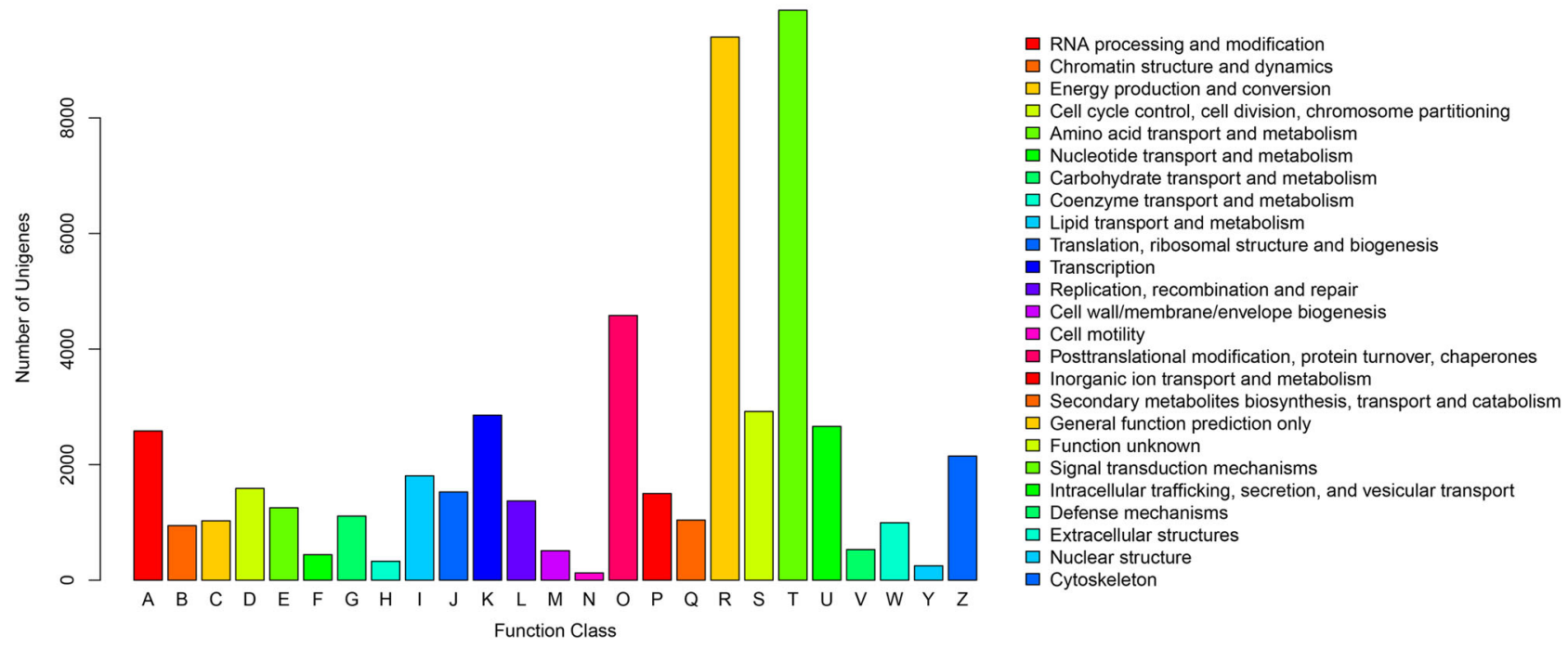

Fig. 3 COG classification for all unigenes

Fig. 4 GO classification for all unigenes. The Blast2GO program was used to obtain GO annotation of all unigenes (level 2). The top $10 \mathrm{GO}$ terms which annotated most from three gene ontology classes, "biological process", "molecular function", and "cell component" were listed respectively

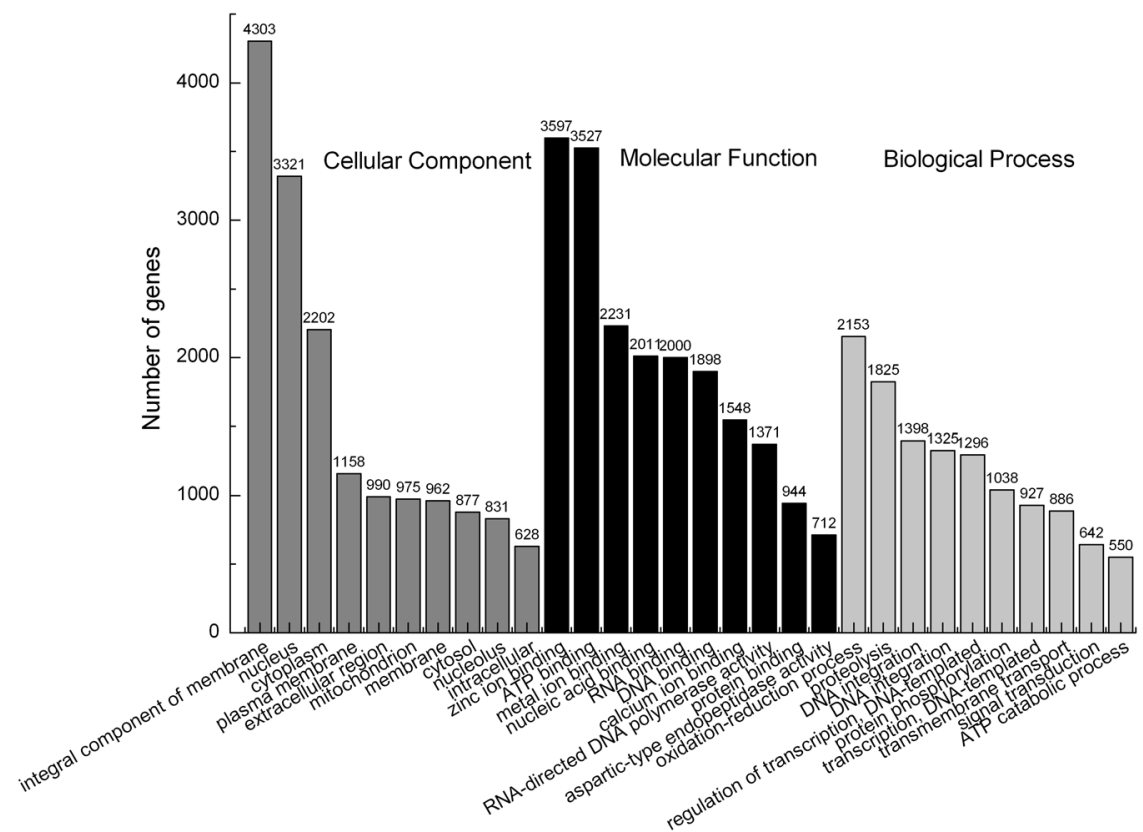

"mitochondrial electron transport" (GO:0006120), mitochondrial membrane"(GO:0031966) (Fig. 5b); 8 GO categories were related to binding, such as "calcium ion binding" (GO:0005509), "iron ion binding" (GO:0005506), "heme binding" (GO:0020037), "copper ion binding" (GO:0005507), and "zinc ion binding" (GO:0008270) (Fig. 5c); 21 GO categories were involved in the regulation of diverse cellular processes, such as "proteolysis" (GO:0006508), "transport" (GO:0006810), "cysteine-type endopeptidase activity" (GO: 0004197) and "peptidase activity" (GO:0008233) (Fig. 5d).

COG classification also presented some categories related to energy metabolism, including "NADH dehydrogenase, subunit 4" (KOG4845), "NADH dehydrogenase subunits 2, 5, and related proteins (KOG4668)", "Cytochrome c oxidase, subunit I" (KOG4769), "Cytochrome c oxidase, subunit II, and related proteins" (KOG4767) and "Cytochrome oxidase subunit III and related proteins" (KOG4664) (Table 2).

\section{qPCR analysis}

Four annotated unigenes (FDR $<0.05)$ that may have connection with the differences in the wolf spider foraging 


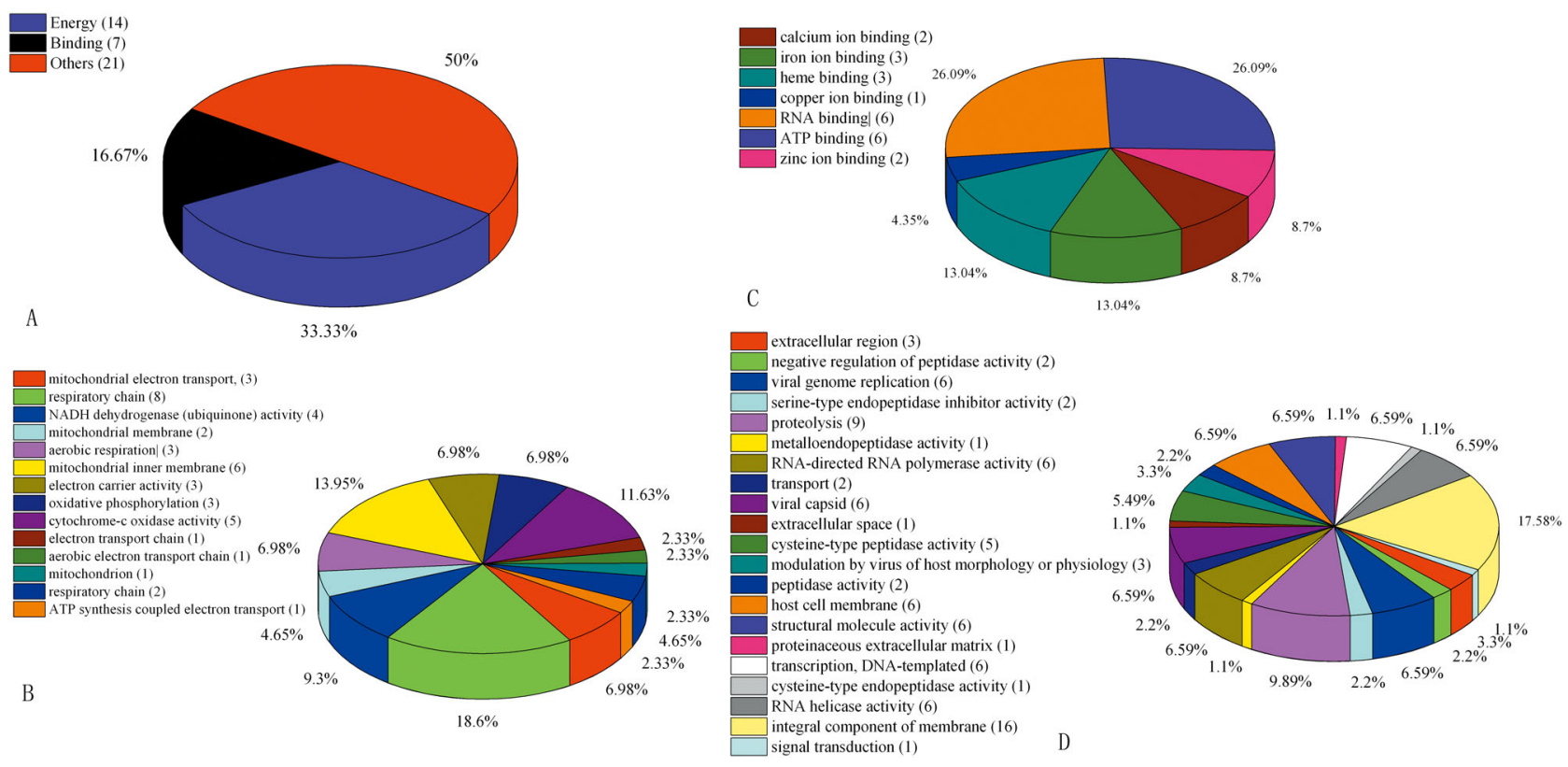

Fig. 5 GO classification for DEGs. A indicates the distribution of total GO terms, B indicates the distribution of terms of regulating energy metabolism, C indicates the distribution of terms of binding, D indicates the distribution of terms of regulation of diverse cellular processes

Table 2 COG classification for DEGs

\begin{tabular}{lll}
\hline KOG.id & KOG.Des & Unigene number \\
\hline KOG4845 & NADH dehydrogenase, subunit 4 & 2 \\
KOG4668 & NADH dehydrogenase subunits 2, 5, and related proteins & 2 \\
KOG4769 & Cytochrome c oxidase, subunit I & 4 \\
KOG4767 & Cytochrome c oxidase, subunit II, and related proteins & 1 \\
KOG4664 & Cytochrome oxidase subunit III and related proteins & 1 \\
KOG3714 & Meprin A metalloprotease & 1 \\
KOG4004 & Matricellular protein Osteonectin/SPARC/BM-40 & 1 \\
KOG1217 & Fibrillins and related proteins containing Ca ${ }^{2+}$-binding EGF-like domains & 1 \\
\hline
\end{tabular}

behavior were selected for $\mathrm{qPCR}$ analysis using primers listed in Table S3.

Uunigene comp16097_c0_seq1, similar to NADH dehydrogenase subunit IV, a key enzyme involved with the respiratory chain, was up-regulated by 1.5 -fold. Unigenes comp76553_c0_seq1, comp50268_c0_seq1 and comp50316_ c0_seq1, all akin to cytochrome oxidase subunit I, an enzyme related with electron transport chain, were up-regulated by 5.1-fold, 1.6-fold, 1.4-fold, respectively (Fig. 6).

\section{Discussion}

Our previous study showed the multi-trophic movement of Cry proteins can be traced from $B t$ rice to BPHs and to a predatory spider (Wang et al. 2016), implying a potential negative influence on safety of predators in the field. In this research, we probed the impacts of $B t$ protein on behavioral ecology like foraging behaviors. Our foraging trial data show that Cry intoxication influenced spider foraging displacement where the movement range was greater than 9 $\mathrm{cm}$. This implied that Cry1 Ab intoxication has the potential to influence the foraging capability of $P$. pseudoannulata. In Apis mellifera, Cry $1 \mathrm{Ab}$ affected food consumption and learning performance, hereby influencing honey bee foraging efficiency (Ramirez-Romero et al. 2008). Based on laboratory experiments with beet armyworms, Spodoptera exugua, Jiang et al. reported that low doses of Cry1Ac protein in an artificial larval diet led to increased long-flight behavior of beet armyworms (Jiang et al. 2013).

Usually, the CNS was a significant regulation core in organisms. In this study, we first conducted CNS transcriptome sequencing of $P$. pseudoannulata, investigating the molecular response of spider to Cry $1 \mathrm{Ab}$. Functional analysis of DEGs indicated that some genes were predicted regulating energy production in mitochondria, such as 

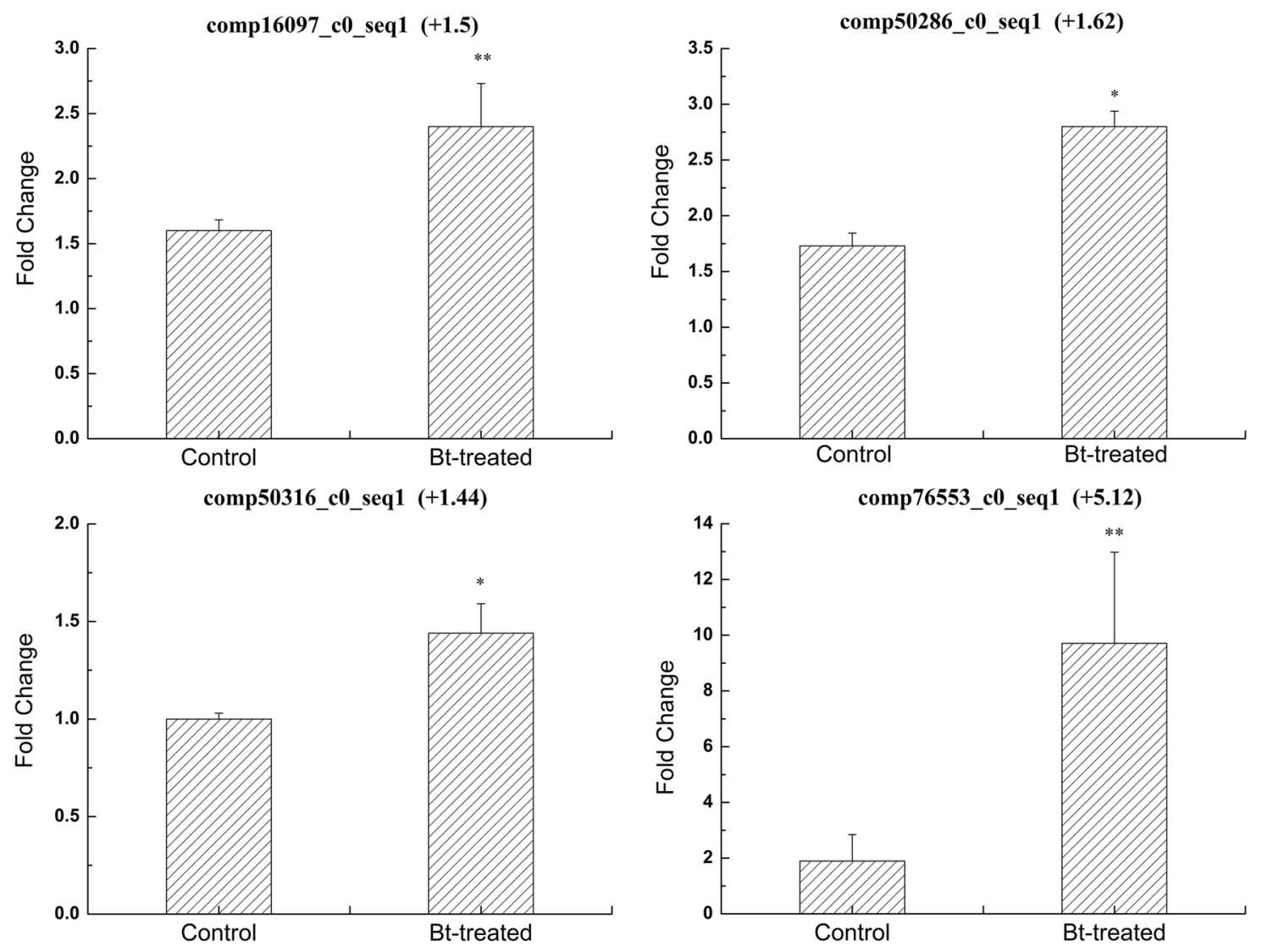

Fig. 6 qPCR analyses of the expression profiles of four annotated DEGs. Three technical replicates were performed for each of three biological replicates. The height of each box represents the mean

"oxidative phosphorylation", "cytochrome-c oxidase activity", "mitochondrial electron transport", and "mitochondrial membrane". These results are similar to that in Tenebrio molitor. The genes expression level involved in mitochondrial respiration was significantly increased in Tenebrio molitor larvae when they intake Cry3Aa protein (Oppert et al. 2012). The mitochondria is a powerhouse in cell, providing energy for various intracellular metabolic reaction (Conley 2016). Obviously, Bt proteins presented a negative impact on energy metabolism in cells. We speculated the changed energy metabolism in the spider may contribute to a more active foraging behavior than controls. On the other hand, some DEGs also have functions related to metal ion binding. Studies suggested that proteins (metalloproteins) contain a metal ion while metals are als required for proteins to carry out their functions (Waldron et al. 2009). These proteins are always acting in protein transportation, enzymes activity and signal transduction (Yi et al. 2009). For example, the iron is a common electron-transfer vector involved in the mitochondrial electron transport chain (Schuler et al. 1999). Hence, some metal ion-dependent reactions may be affected in response to Cry $1 \mathrm{Ab}$ in CNS of

average of sample-specific 2-DCt values. Bars displayed mean $+\mathrm{SD}$ and asterisks $(*)(* *)$ in the table are significantly different (One-way ANOVA, $p<0.05, p<0.01)$

P. pseudoannulata. This may be associated with a Cry $1 \mathrm{Ab}$ tolerance mechanism in the spider.

Acknowledgements The experiment was supported by the National Natural Science Foundation of China (No. 31071943, No. 31272339, No. 31472017, No. 41401358), China Postdoctoral Science Foundation (No. 2013M542112), and the Agricultural Science and Technology Innovation Program of China (No. CAAS-ASTIP-IBFC).

\section{Compliance with ethical standards}

Conflict of interest The authors declare that they have no competing interests.

Ethical approval Materials used in this study were unregulated common arthropod spider and BPHs.

Open Access This article is distributed under the terms of the Creative Commons Attribution 4.0 International License (http://crea tivecommons.org/licenses/by/4.0/), which permits unrestricted use, distribution, and reproduction in any medium, provided you give appropriate credit to the original author(s) and the source, provide a link to the Creative Commons license, and indicate if changes were made. 


\section{References}

Ashburner M, Ball CA, Blake JA, Botstein D, Butler H, Cherry JM, Davis AP, Dolinski K, Dwight SS, Eppig JT, Harris MA, Hill DP, Issel-Tarver L, Kasarskis A, Lewis S, Matese JC, Richardson JE, Ringwald M, Rubin GM, Sherlock G (2000) Gene ontology: tool for the unification of biology. The Gene Ontology Consortium. Nat Genet 25:25-29. https://doi.org/10.1038/75556

Akhtar ZR, Tian JC, Chen Y et al. (2010) Impacts of six bt rice lines on nontarget rice feeding thrips under laboratory and field conditions. Environ Entomol 39:715-726

Audic S, Claverie JM (1997) The significance of digital gene expression profiles. Genome Res 7:986-995

Bottrell DGSK (2012) Resurrecting the ghost of green revolutions past: The brown planthopper as a recurring threat to high-yielding rice production in tropical Asia. J Asia Pac Entomol 15:122-140

Chen M, Shelton A, Ye GY (2011) Insect-resistant genetically modified rice in China: from research to commercialization. Annu Rev Entomol 56:81-101

Chen M, Ye GY, Liu ZC et al. (2009) Analysis of Cry1Ab toxin bioaccumulation in a food chain of $\mathrm{Bt}$ rice, an herbivore and a predator. Ecotoxicology 18:230-238

Conley KE (2016) Mitochondria to motion: optimizing oxidative phosphorylation to improve exercise performance. J Exp Biol. 219:243-249

Duelli POM (1998) In search of the best correlates for local organismal biodiversity in cultivated areas. Biodivers Conserv. 7:297-309

Jung M-PS-TK, Hunsung K, Lee J-H (2008a) Biodiversity and community structure of ground-dwelling spiders in four different field margin types of agricultural landscape in Korea. Appl Soil Ecol. 38:185-195

Jiang XF, Chen J, Zhang L, Sappington TW, Luo LZ (2013) Increased long-flight activity triggered in beet armyworm by larval feeding on diet containing Cry1Ac protoxin. PLoS ONE 8:e63554

Khush GS (2005) What it will take to feed 5.0 billion rice consumers in 2030. Plant Biotechnol J 59:1-6

Lee SY, Kim ST, Jung JK, Lee JH (2014) A comparison of spider communities in Bt and non-Bt rice fields. Environ Entomol 43:819-827

Livak KJ, Schmittgen TD (2001) Analysis of relative gene expression data using real-time quantitative PCR and the 2(-Delta Delta C (T)). Method. Methods 25:402-408

Lu ZB, Tian JC, Wang W et al. (2014) Impacts of Bt rice expressing Cry1C or Cry2A protein on the performance of nontarget leafhopper, Nephotettix cincticeps (Hemiptera: Cicadellidae), under laboratory and field conditions. Environ Entomol 43:209-217
Marc PCA, Ysnel F (1999) Spiders (Aranea) useful for pest limitation and bioindication. Agric Ecosyst Environ 74:229-273

Mortazavi A, Williams BA, McCue K, Schaeffer L, Wold B (2008) Mapping and quantifying mammalian transcriptomes by RNASeq. Nat Methods 5:621-628

Oppert B, Dowd SE, Bouffard P, Li L, Conesa A, Lorenzen MD, Toutges M, Marshall J, Huestis DL, Fabrick J, Oppert C, JuratFuentes JL (2012) Transcriptome profiling of the intoxication response of Tenebrio molitor larvae to Bacillus thuringiensis Cry3Aa protoxin. PLoS ONE 7:e34624. https://doi.org/10.1371/ journal.pone.0034624

Ouyang S, Liu J, Jones KM, et al (2005) The map-based sequence of rice genome. Nature 436:793-800

Ramirez-Romero R, Desneux N, Decourtye A, Chaffiol A, PhamDelegue MH (2008) Does Cry1Ab protein affect learning performances of the honey bee Apis mellifera L. (Hymenoptera, Apidae). Ecotoxicol Environ Saf 70:327-333

Sauberer NZK, Abensperg-Traun M, Berg H-M, Bieringer G, Milasowszky NMD, Plutzar C, Pollheimer M, Storch C, Trostl RZH, Grabherr G (2004) Surrogate taxa for biodiversity in agricultural landscapes of eastern Austria. Biol Conserv 117:181-191

Schuler F, Yano T, Di Bernardo S, Yagi T, Yankovskaya V, Singer TP, Casida JE (1999) NADH-quinone oxidoreductase: PSST subunit couples electron transfer from iron-sulfur cluster N2 to quinone. Proc Natl Acad Sci USA. 96:4149-4153

Tian JC, Chen Y, Li ZL et al. (2012) Transgenic Cry1Ab rice does not impact ecological fitness and predation of a generalist spider. PLoS ONE 7:e35164

Tian YX, Zhou Y, Xiao KF, Wang Z, CJ J et al. (2013) Effect of $\mathrm{Cry} 1 \mathrm{Ab}$ protein on hemocytes of the wolf spider Pardosa pseudoannulata. Biocontrol Sci.Tech 23:423-432

Wang J, Peng YD, Chao $\mathrm{H}$ et al. (2016) Cry1 Ab-expressing rice did not influence expression of fecundity-related genes in the wolf spider Pardosa pseudoannulata. Gene 592(1):1-7

Waldron KJ, Rutherford JC, Dianne F, Robinson NJ (2009) Metalloproteins and metal sensing. Nature 460:823-830

Yi L, Natasha Y, Nathan S, Marshall NM (2009) Design of functional metalloproteins. Nature 460:855-862

Zhao WCCJ, Chen ZX (2004) Development of a monoclonal antibody to detect predation of the brown planthopper Nilaparvata lugens (Stål). Int J Pest Manag. 50:317-321

Zhao WCCJ, Zhang WJ (2005) Evaluation of the control effects of Pardosa pseudoannulata on Nilaparvata lugens (Sta 1) with amonoclonal antibody. Acta Ecol Sin 25:78-82 